\title{
Island, pit and groove formation in strained heteroepitaxy
}

\author{
M.T. Lung ${ }^{1}$, Chi-Hang Lam ${ }^{1}$, and Leonard M. Sander ${ }^{2}$ \\ ${ }^{1}$ Department of Applied Physics, Hong Kong Polytechnic University, Hung Hom, Hong Kong, China \\ ${ }^{2}$ Michigan Center for Theoretical Physics, Department of Physics, \\ Randall Laboratory, University of Michigan, Ann Arbor, MI 48109-1120, USA
}

(Dated: September 28, 2018)

\begin{abstract}
We study the morphological evolution of strained heteroepitaxial films using a kinetic Monte Carlo method in three dimensions. The elastic part of the problem uses a Green's function method. Isolated islands are observed under deposition conditions for deposition rates slow compared with intrinsic surface roughening rates. They are semi-spherical and truncated conical for high and low temperature cases respectively. Annealing of films at high temperature leads to the formation of closely packed islands consistent with an instability theory. At low temperature, pits form via a layer-by-layer nucleation mechanism and subsequently develop into grooves.
\end{abstract}

PACS numbers: 68.65.-k, 68.65.Hb, 81.16.Dn, 81.16.Rf

Epitaxial growth techniques have been used to deposit strained coherent films on substrates of a different materials with a mismatched lattice constant. This is called heteroepitaxy. Many experiments have shown that beyond a threshold film thickness, an array of three dimensional (3D) nanosized islands self-assembles under favorable growth conditions [1, 2, 3]. These results are of considerable interest since the islands behave as quantum dots and are expected to find applications in future microelectronic devices. The most intensively studied examples include $\mathrm{Ge} / \mathrm{Si}(100)$ and more generally its alloy variant $\mathrm{Si}_{1-x} \mathrm{Ge}_{x} / \mathrm{Si}(100)$ [4, 5, 6, 6, 8]. The island morphology depends strongly and often non-trivially on the lattice misfit dictated by the Ge concentration as well as growth conditions including temperature and deposition rate. In addition, other interesting nanostructures including 3D pits, grooves and quantum dot molecules composed of coupled islands and pits are also generated under appropriate conditions 9, 10].

In this letter, we report large scale 3D kinetic Monte Carlo simulations on the morphological evolution of strained layers. Our simulations generate morphologies very reminiscent of those observed under various growth or annealing conditions. We should note that the simulation of strained layers is computationally challenging due to the long range nature of elastic interactions. Previous atomistic simulations are limited to two dimensions (2D) 11, 12, 13, 14] or sub-monolayer coverage 15]. Continuum computations are less difficult but cannot reliably account for faceted surfaces and fluctuations which are especially important at the early stage of roughening 16, 17, 18].

We model the film and substrate system by a simple cubic lattice of balls and springs $11,12,13,15$. The substrate consists of $64 \times 64 \times 64$ atoms. Periodic boundary conditions in lateral directions and fixed boundary conditions for the bottom layer are assumed. The substrate has a lattice constant $a_{s}=2.72 \AA$ which gives an atomic density appropriate for crystalline silicon. The lattice constant $a_{f}$ of the film is related to the lattice misfit $\epsilon=\left(a_{f}-a_{s}\right) / a_{f}$. Nearest neighboring $(\mathrm{NN})$ and next nearest neighboring $(\mathrm{NNN})$ atoms are directly connected by elastic springs with force constants $k_{1}=2 \mathrm{eV} / \mathrm{a}_{s}^{2}$ and $k_{2}=k_{1}$ respectively. The elastic couplings of adatoms with the rest of the system are weak and are completely neglected.

Our algorithm imposes solid-on-solid conditions with atomic steps limited to at most one atom high. Every topmost atom in the film can hop to a different random topmost site within a neighborhood of $l \times l$ columns with equal probability. We put $l=33$. Decreasing the hopping range does not alter our results significantly. The hopping rate $\Gamma_{m}$ of a topmost atom $m$ follows an Arrhenius form

$$
\Gamma_{m}=R_{0} \exp \left[-\frac{n_{1 m} \gamma_{1}+n_{2 m} \gamma_{2}-\Delta E_{m}-E_{0}}{k_{B} T}\right]
$$

Here, $n_{1 m}$ and $n_{2 m}$ are the number of NN and NNN of atom $m$ respectively while $\gamma_{1}=0.085 \mathrm{eV}$ and $\gamma_{2}=\gamma_{1} / 2$ are the corresponding bond strengths. The elastic energy of the hopping atom is denoted by $\Delta E_{m}$ and will be explained later. Finally, we put $E_{0}=0.415 \mathrm{eV}$ and $R_{0}=$ $2 D_{0} /\left(\sigma a_{s}\right)^{2}$ with $D_{0}=4.1 \times 10^{13} \AA^{2} s^{-1}$ and $\sigma^{2}=l^{2} / 6$. This gives the appropriate adatom diffusion coefficient for silicon (100) 19]. Our choice of the ratios $k_{1} / k_{2}$ and $\gamma_{1} / \gamma_{2}$ maximizes the isotropy of the system.

The elastic energy, $\Delta E_{m}$, has to be repeatedly calculated during a simulation; this dominates the CPU time. $\Delta E_{m}$ is defined as the difference in the strain energy $E_{s}$ of the whole lattice at mechanical equilibrium when the site is occupied minus that when it is unoccupied. Calculating $E_{s}$ requires solving a long-range elasticity problem to obtain the atomic positions of every atom in the film and the substrate. We have found it possible to significantly speed up the calculation by appling an exact Green's function method. A method of this type was introduced by Tewary [20] in the context of point impurities. We generalized the technique to free surfaces in Ref. [13]. The result of these developments is that we can solve the elastic problem at a surface site using reduced equations involving only other surface atoms. Moreover, we use a surface coarsening scheme in which morphological 

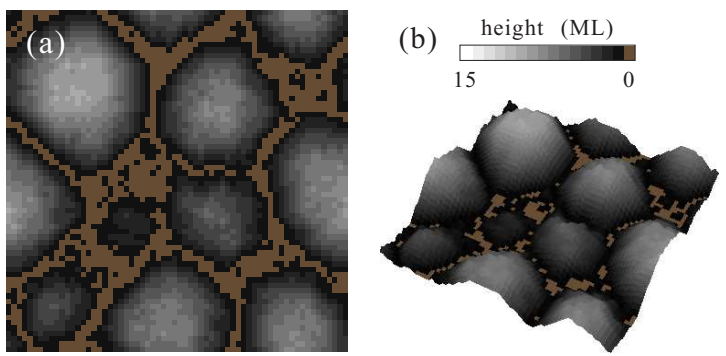

FIG. 1: Surface from simulation of deposition at $1000 \mathrm{~K}$ and $20000 \mathrm{MLs}^{-1}$ in top view (a) and 3D view (b). The gray scale shows the local height of the surface and the exposed part of the substrate is shaded in brown.

details of the surface far away from atom $m$ are averaged 13. As a result, calculating $\Delta E_{m}$ involves only about 160 effective particles and takes less than one second on a $3 \mathrm{GHz}$ pentium computer. Hopping events are then sampled using an acceptance-rejection algorithm aided by quick estimates of $\Delta E_{m}$ which enables a high acceptance probability. A simulation reported here typically involves $10^{6}$ successful hopping events and takes 10 days to complete. We have considered large misfit and in some cases also high deposition rate so that the computations can be manageable.

We have simulated deposition of films with $8 \%$ lattice misfit at temperature $1000 \mathrm{~K}$ and deposition rate 20000 $\mathrm{ML}^{-1}$. Figure 1 shows the resulting morphology from a typical run at a nominal film thickness of 3MLs. Isolated semi-spherical islands are observed. Most of them nucleate when the nominal coverage is about $1 \mathrm{ML}$ and then grow steadily as more atoms are deposited. Coarsening via exchange of atoms among islands (Ostwald ripening) also occurs. Some small islands shrink and vanish eventually. However, coalescence of islands is suppressed by their mutual elastic repulsion 21]. In fact, the edges of neighboring islands are often deformed to avoid each others.

In our simulations, as in experiment, the deposition rate has a substantial effect on surface morphology. At the rate considered above, island growth is limited by the supply of atoms. Individual islands have already relaxed to their equilibrium shapes. That is, deposition is slow relative to the formation dynamics and geometrical relaxation of islands. In contrast, at lower deposition rates, we observe that islands become larger and less dense because there is more time for coarsening. For deposition faster than island formation, layers of atoms quickly accumulate before the resulting film roughens 13 . With an abundant supply of atoms, we observe that the roughening dynamics is similar to that for annealing except for a trivial vertical drift of the whole surface. We will discuss annealing next.

We have simulated annealing of initially flat films with $10 \mathrm{MLs}$ of atoms and $6 \%$ lattice misfit at 1000K. Figures 2(a)-(c) show snapshots of the evolution. 2D islands and pits first develop leading to a high step density [Fig.
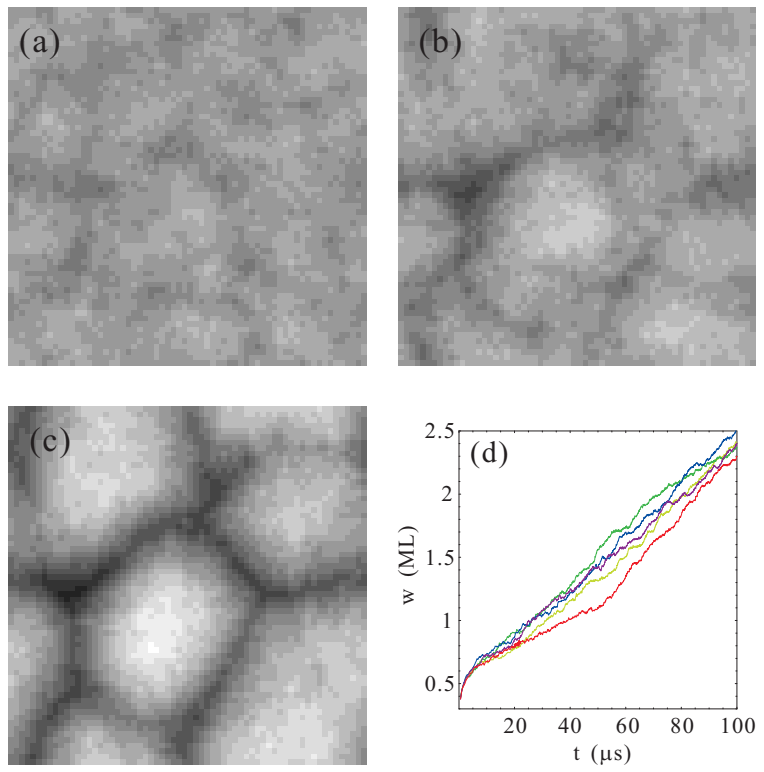

FIG. 2: Snapshots from annealing of an initially flat film at $1000 \mathrm{~K}$ at time $t=20$ (a), 50 (b) and $100 \mu \mathrm{s}$ (c), and a plot of surface width $w$ against $t$ for 5 independent runs (d).

2(a)]. At this point, the film is still relatively flat and highly stressed. The misfit has little impact on the morphology except for an enhancement of the step density due to a reduction of the effective step free energy. As the roughness increases, long-range elastic interactions begin to dominate and lead to the formation of 3D islands and pits with gentle slopes [Fig. 2(b)]. Subsequently, well developed 3D islands bounded by a network of grooves emerge [Fig. 2(c)]. Note that the surface inclination at many grooves has reached its maximum value allowed in our model. In experiments on the $\mathrm{Si}-\mathrm{Ge}$ system, grooves are often bounded by [115] facets. The physical reasons for this might be the same, though our model is too crude to select among facets.

The (100) surface studied above does not act like a true facet as is evident from the abundance of surface steps in Fig. 2(a). This indicates that $1000 \mathrm{~K}$ is above the surface roughening transition temperature. Thus, the surface energy varies smoothly with the local inclination. In this situation, the strain-induced roughening of an unfaceted surface should be described by the Asaro-Tiller-Grinfeld instability theory 22] which predicts that random perturbations of the surface at sufficiently long wavelength spontaneously amplify. The surface will gradually be dominated by modulations at the most unstable wavelengths.

Our annealing results at $1000 \mathrm{~K}$ are consistent with the instability theory. This is supported by a few characteristic features. First, the sidewalls of the newly emerging islands are gentle and their inclinations increase gradually rather than abruptly. Moreover, the island base areas stay relatively constant. As a further evidence, Fig. 2(d) plots the r.m.s. surface width $w$ against the annealing 

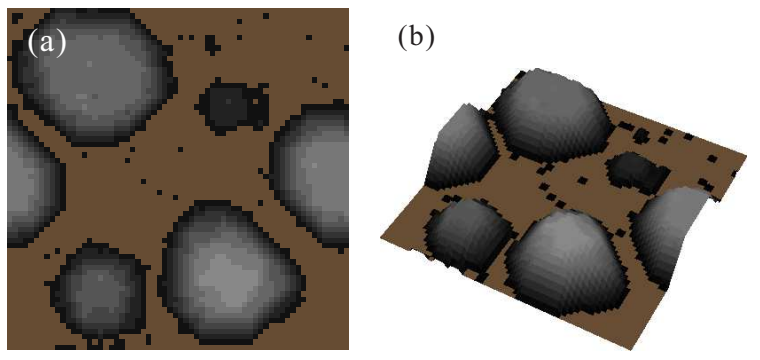

FIG. 3: Surface from simulation of deposition at $600 \mathrm{~K}$ and $10 \mathrm{ML}^{-1}$.
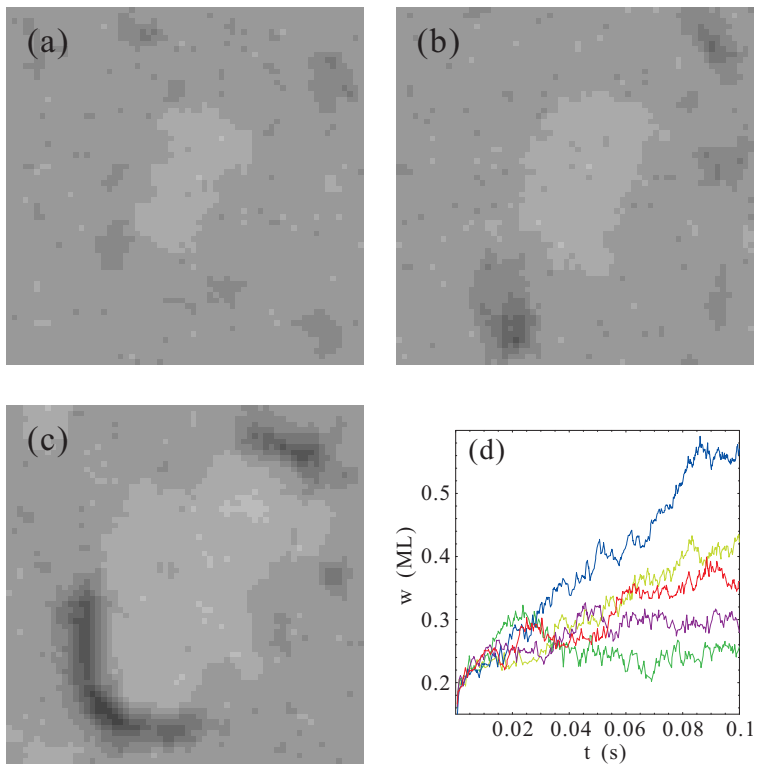

FIG. 4: Snapshots from annealing of an initially flat film at $600 \mathrm{~K}$ at time $t=0.1$ (a), $0.15(\mathrm{~b})$ and $0.22 \mathrm{~s}(\mathrm{c})$, and a plot of $w$ against $t$ for 5 independent runs (d).

time $t$ for 5 independent runs. We observe that $w$ increases steadily and the ensemble fluctuations are small as expected for barrierless processes. The morphological development also qualitatively resembles the initial evolution of $\mathrm{Si}_{1-x} \mathrm{Ge}_{x} / \mathrm{Si}(100)$ films at high temperature and low misfit [7]. Tersoff, et. al have argued that the $\mathrm{Si}_{1-x} \mathrm{Ge}_{x}(100)$ surface under these conditions is not a true facet [23] and theories based on unfaceted surfaces should apply.

Next, we consider a lower temperature, 600K, which gives drastically different morphologies indicating distinct roughening mechanisms. Figure 3 shows a surface at a nominal coverage of $2 \mathrm{MLs}$ from a simulation of deposition at $8 \%$ misfit and $10 \mathrm{ML} \mathrm{s}^{-1}$. We again observe isolated islands but they now take the shapes of truncated cones. Most islands are out of equilibrium as their heights are clearly limited by significant energy barriers for upper layer nucleation.

We have also simulated annealing at $600 \mathrm{~K}$. Figures 4(a)-(c) show three snapshots from a typical run. A large 2D island and a few smaller 2D pits first appear
[Fig. 4(a)]. Later, 3D pits develop [Fig. 4(b)]. They then become increasingly eccentric and gradually turn into grooves [Fig. 4(c)]. Analogous 3D structures are also observed for deposition at rates fast compared to roughening.

It is interesting to note that only part of the surface roughens even after a long annealing time in sharp contrast to the high temperature case. This strongly suggests that the surface is a true facet and $600 \mathrm{~K}$ is below the roughening temperature. The surface energy should be a singular function of the slope and instability theory should not apply. For this situation, a nucleation theory has been suggested for 3D island or pit formation on faceted surfaces [24, 25]. According to this approach, an island or pit has to overcome an energy barrier associated with a critical volume before it can be stable. Experiments on island formation at low temperature and high misfit have indicated better agreement with nucleation theory [24].

Figure 4 (d) plots the r.m.s. surface width $w$ against time from 5 independent runs during early stage of roughening. There are large ensemble fluctuations supporting the relevance of nucleation processes. However, there exists no dominating jump in $w$ associated with a single successful nucleation event after which $w$ grows steadily. Instead, multiple relatively rapid increments can be observed and are associated with the creation of lower layers in the dominant pits. Conventional nucleation theory does not successfully describe the effects of the large barriers for nucleation of further layers. The formation of 3D pits in Figs. 4(a)-(c) and in fact also of the 3D islands in Fig. 3 should best be described by a sequence of layer-by-layer nucleation events. For a growing pit for instance, atoms are ejected continuously while lateral expansion takes place at constant pit depth. Once the bottom becomes sufficiently large, nucleation of a further layer will be possible. The growth is thus based on the correlated processes of continuous lateral expansion and periodic sudden nucleation of deeper layers. The associated rates depend not only on the pit geometry but also on the presence of nearby islands or pits due to both exchange of atoms and elastic interactions. Recent theories on the elasticity of step mounds should be particularly relevant for further analysis [26, 27].

The selection mechanism between islands and pits also deserves further explanation. Continuum elasticity theory shows that islands and pits with infinitesimal slopes relieve elastic energy equally well [24]. It is visually apparent that an up-down symmetry exists for surfaces in Fig. 2(a) and to a lesser extent also in Fig. 2(b). However, pits are increasingly favored energetically compared to islands as local slopes become steeper [28]. At low temperatures, the energy difference is already significant for single layered structures. Specifically, asymmetry between 2D islands and pits is already apparent in Fig. 4(a). There is typically one dominant island but a few smaller pits. This is because the lower energy of pits also implies a lower nucleation barrier. Pits can hence 
nucleate more quickly and are more abundant. Because new islands are not nucleated, the existing one absorbs all ejected atoms and grows quickly. Furthermore, the better stability of pits also explains the development of $3 \mathrm{D}$ pits rather than 3D islands in Figs. 4(b)-(c). We have observed 3D islands only in Fig. 3] for slow deposition. This is because under this slow deposition rate, 3D islands are already able to develop before a thick enough film can be formed to accommodate the pits [13]. Experimentally, the selected structure also turns from 3D islands to $3 \mathrm{D}$ pits upon lowering the temperature and increasing the deposition rate [9].

An interesting transition from pits to grooves is also observed in Figs. 4(b)-(c). For shallow pits, a square base is energetically preferred to a rectangular one [24]. This explains the more rounded shapes of the pits in Fig. 4(b). As the pits enlarge, their sidewalls also become steeper to relieve the stress more efficiently. Grooves are then energetically preferred to rounded pits because their linear extents are larger and can lead to stress relief over a much wider region. Formation of grooves in Fig. 4(c) is further enhanced by the stress around a $2 \mathrm{D}$ island. This is closely related to the phenomena of cooperative nucleation [5] and also trench formation around 3D islands [29]. The presence of a neighboring island however is not essential as we have also observed pits turning into grooves far away from any islands. Grooves are also observed in experiments from annealing of pits [10].
In conclusion, we have applied a kinetic Monte Carlo method in 3D to study morphological structures generated from deposition and annealing of strained heteroepitaxy. Under deposition conditions, morphologies depend dramatically on whether deposition is slow compared to the intrinsic roughening rate of the surface as in the $2 \mathrm{D}$ case [13]. For slow deposition, isolated islands result and their formation and development are limited by the supply of atoms. In contrast during fast deposition, 3D structures form only after layers of atoms have accumulated and are similar to those from annealing of initially flat films. Morphologies from annealing further show strong dependence on temperature which determines whether the initial surface is faceted. Upon annealing at high temperature, unfaceted surfaces develop arrays of 3D islands via the Asaro-Tiller-Grinfield instability. In contrast, faceted surfaces at low temperature develop 3D pits via a layer-by-layer nucleation mechanism. The pits later turn into grooves. We suggest that the selection mechanisms between islands and pits as well as between pits and grooves are of energetic origin. Many of the general trends that we observe in our simulations are similar to experimental results.

We thank J.A. Floro for helpful discussions. This work was supported by HK RGC, Grant No. PolyU-5289/02P. LMS is supported in part by NSF grant No. DMS0244419 .
[1] V.A. Shchukin and D. Bimberg, Rev. Mod. Phys. 71, 1125 (1999).

[2] P. Politi et al, Phys. Rep. 324, 271 (2000).

[3] L.B. Freund and S. Suresh, Thin film materials, stress, defect formation and surface evolution (Cambridge University Press, 2003).

[4] Y.-W. Mo, D.E. Savage, B.S. Swartzentruber, and M.G. Lagally, Phys. Rev. Lett. 65, 1020 (1990).

[5] D.E. Jesson, K.M. Chen, S.J. Pennycook, T. Thundat, and R.J. Warmack, Phys. Rev. Lett. 771330 (1996).

[6] A. Vailionis et al, Phys. Rev. Lett. 85, 3672 (2000).

[7] J.A. Floro, E. Chason, L.B. Freund, R.D. Twesten, R.Q. Hwang, and G.A. Lucadamo, Phys. Rev. B 59, 1990 (1999).

[8] P. Sutter, P. Zahl, and E. Sutter, Appl. Phys. Lett. 82, 3454 (2003).

[9] J.L. Gray, R. Hull, J.A. Floro, Appl. Phys. Lett. 81, 2445 (2002).

[10] J.L. Gray, R. Hull, J.A. Floro, Appl. Phys. Lett. 85, 3253 (2004).

[11] B.G. Orr, D.A. Kessler, C.W. Snyder, and L.M. Sander, Euro. Phys. Lett., 19, 33 (1992).

[12] K.E. Khor and S. Das Sarma, Phys. Rev. B 62, 16657 (2000).

[13] C.H. Lam, C.K. Lee, and L.M. Sander, Phys. Rev. Lett. 89, 216102 (2002).

[14] F. Much and M. Biehl, Euro. Phys. Lett. 63, 14 (2003).

[15] M. Meixner, E. Schöll, V.A. Shchukin, and D. Bimberg, Phys. Rev. Lett. 87, 236101 (2001)
[16] W.H. Yang and D.J. Srolovitz, Phys. Rev. Lett. 71, 1593 (1993);

[17] Y.W. Zhang, A.F. Bower, and P. Liu, Thin Solid Films 424, 9 (2003).

[18] A. Ramasubramaniam and V.B. Shenoy, J. Appl. Phys. 89, 7813 (2004).

[19] D.E. Savage et al, in Semiconductors and Semimetals 56, R. Hull and J.C. Bean Ed. (Academic Press 1999).

[20] V.K. Tewary, Adv. Phys. 22, 757 (1973).

[21] D.E. Jesson, T.P. Munt, V.A. Shchukin, and D. Bimberg, Phys. Rev. B 69, 041302 (2004).

[22] M.A. Grinfeld, J. Nonlinear Sci. 3, 35 (1993).

[23] J. Tersoff, B.J. Spencer, A. Rastelli, and H. von Känel, Phys. Rev. Lett. 89, 196104 (2002).

[24] J. Tersoff and F.K. LeGoues, Phys. Rev. Lett. 72, 3570 (1994).

[25] M. Bouville, J.M. Millunchick and M.L. Falk, unpublished.

[26] V.M. Kaganer and K.H. Ploog, Phys. Rev B 64, 205301 (2001).

[27] V.B. Shenoy and L.B. Freund, J. Mech. Phys. Solids 50, 1817 (2002).

[28] D. Vanderbilt and L.K. Wickham, in Evolution of ThinFilm and Surface Microstructure, C.V. Thompson et. al Ed., MRS Symposia Proceedings No. 202 (MRS, Pittsburgh, 1991), p.555.

[29] S.A. Chaparro, Y. Zhang, and J. Drucker, Appl. Phys. Lett. 76, 3534 (2000). 